\title{
Síndrome metabólica em uma fábrica de papel no Estado do Paraná
}

\author{
Metabolic syndrome in a paper factory in the State of Paraná, Brazil
}

\author{
Caími Tibiriçá de Carvalho'
}

\begin{abstract}
RESUMO | Contexto: A síndrome metabólica (SM) tem grande importância para identificação precoce de eventos cardiovasculares. O conhecimento acerca dessa manifestação ainda é escasso, especialmente no âmbito laboral. Objetivo: Identificar colaboradores com SM durante o exame periódico do ano de 2015, de acordo com o sexo e a idade. Métodos: Foi realizado estudo transversal durante o exame periódico de 2015, em que foram avaliados 1.787 colaboradores de uma fábrica de papel no Estado do Paraná, sendo 1.643 homens e 144 mulheres com idades entre 21 e 65 anos. Os colaboradores compareceram ao ambulatório médico com resultado dos exames laboratoriais como glicemia de jejum, colesterol total e frações, e triglicerídeos. Também foram aferidas a pressão arterial, a medida do peso e a altura para o cálculo do índice de massa corporal (IMC) e para a medida da circunferência abdominal. A SM foi definida segundo critério estabelecido pela International Diabetes Federation (IDF). Foi utilizado o teste do $\chi^{2}$ para avaliar a incidência de SM. Resultados: Constatou-se que 16,9\% dos colaboradores da fábrica têm SM. Entre os homens, a porcentagem de SM foi de 17,9\%, e entre as mulheres, de 5,5\%. Conclusão: Esses dados mostram a importância de implantação de Programas de Qualidade de Vida visando reduzir ou prevenir fatores de risco e a ocorrência dos agravos. Esses programas promovem a melhoria da saúde em médio e longo prazo, o que proporciona melhores condições de trabalho e de vida.
\end{abstract}

Palavras-chave I incidência; medicina do trabalho; qualidade de vida; índice de massa corporal; circunferência abdominal.

ABSTRACT | Context: Metabolic syndrome (MS) is highly important to the early identification of cardiovascular events. However, knowledge on this manifestation is still rare, especially at the working field. Objective: To identify workers with MS during the 2015 periodic examination according to sex and age. Methods: A cross-sectional study during the 2015 periodic examination was carried out. It evaluated 1,787 workers from a paper factory in the state of Paraná, Brazil (1,643 men and 144 women aged 21 to 65 years). Workers attended the medical outpatient clinic bearing their results of laboratorial examinations, such as fasting blood glucose test, total cholesterol and fractions, and triglycerides. Blood pressure, weight, and height were measured to calculate the body mass index (BMI) and abdominal circumference measurements. The MS was established based on criteria from the International Diabetes Federation (IDF). The $\chi^{2}$ test was used for assessing the MS incidence. Results: It was found that $16.9 \%$ of the factory workers have MS. The MS percentage among men was of $17.9 \%$ and $5.5 \%$ among women. Conclusion: These data show the importance of implementing Quality of Life Programs that aim at reducing or preventing risk factors and damage occurrence. These programs promote health improvement at medium and long terms, which provide better work and life conditions.

Keywords I incidence; occupational medicine; quality of life; body mass index; abdominal circumference. 


\section{INTRODUÇÃO}

As mudanças no modo de vida que a sociedade tem vivenciado nas últimas décadas, como a tecnologia da informação e automação, têm impacto direto no processo saúdedoença, especialmente no âmbito laboral, desencadeando uma série de doenças relacionadas ao aumento da jornada de trabalho, ao isolamento social, aos elevados níveis de estresse, ao consumo excessivo de calorias e de gorduras saturadas e aos estilos de vida sedentários, prejudicando o ambiente de trabalho e o relacionamento social e familiar $^{1}$. Todos esses fatores contribuem para uma epidemia de doenças crônicas, como obesidade, diabetes mellitus e hipertensão arterial, condições que frequentemente cursam com alterações lipídicas que aumentam o risco de eventos cardiovasculares ${ }^{1}$.

A síndrome metabólica (SM) tem grande importância para a identificação precoce dos eventos cardiovasculares, já que está diretamente relacionada aos fatores de risco ${ }^{2}$. Ela não é uma doença específica, mas sim uma série de fatores de risco de origem metabólica, como obesidade abdominal, triglicerídeos elevados, HDL baixo, intolerância à glicose e hipertensão. $\mathrm{O}$ conhecimento acerca da SM ainda é escasso na população em geral e principalmente em trabalhadores, uma vez que eles permanecem a maior parte do dia no local de trabalho, alimentando-se nele ou em suas proximidades. A qualidade e a duração do sono podem contribuir para o surgimento da SM ou a alteração da função endócrina ${ }^{1}$.

Apesar de o conceito estar bem definido, os critérios para o diagnóstico que foram definidos por diversas organizações, como a Organização Mundial de Saúde (OMS), o Grupo Europeu para o Estudo da Resistência a Insulina (EGIR), a International Diabetes Federation (IDF), o National Cholesterol Education Program Third Adult Treatment Panel (NCEP - ATPIII) e a Associação Americana da Diabetes (ADA), não têm sido coincidentes. A principal diferença entre o IDF e o NCEP - ATPIII está no valor limite do perímetro abdominal. A SM, neste trabalho, foi definida segundo critério estabelecido pela IDF: circunferência abdominal em homens maior ou igual a $94 \mathrm{~cm}$ e em mulheres maior ou igual a $80 \mathrm{~cm}$, associada à presença de 2 ou mais componentes que se seguem: nível de triglicerídeos maior ou igual a $150 \mathrm{mg} / \mathrm{dL}, \mathrm{HDL}$ menor que $40 \mathrm{mg} / \mathrm{dL}$ em homens e menor que $50 \mathrm{mg} / \mathrm{dL}$ em mulheres, pressão arterial maior ou igual a $130 / 85 \mathrm{mmHg}$ e glicemia em jejum maior ou igual a $100 \mathrm{mg} / \mathrm{dL}$, tanto para homens quanto para mulheres ${ }^{2}$.

A patogênese da SM é multifatorial, sendo o sedentarismo, a idade avançada, o estresse emocional, as modificações hormonais e os determinantes genéticos os principais fatores desencadeantes. A prevalência de SM está associada à excessiva deposição de gordura abdominal e é maior entre indivíduos pertencentes às classes socioeconômicas mais baixas $^{3}$. Estima-se que, na maioria dos países, entre $20 \mathrm{e}$ $30 \%$ da população adulta tenha $\mathrm{SM}^{2}$.

Para as empresas é de grande importância identificar esse perfil, pois, além de aumentar os custos causados pelas doenças crônicas e licenças médicas dos trabalhadores afetados por tal condição, existe a diminuição da produtividade, visto que esses colaboradores podem ter uma limitação física associada à obesidade, ao diabetes ou à hipertensão ${ }^{4}$.

Mesmo sendo de expressiva relevância, não há, até o presente momento, estudos específicos sobre SM de trabalhadores em ambientes fabris no Brasil. A identificação dessa síndrome durante a avaliação periódica de saúde permite alertar os acometidos sobre o maior risco de adoecimento e evitar afastamentos do trabalho, limitações laborais e até aposentadorias precoces, propondo modificações de estilo de vida, reeducação de hábitos e até intervenções medicamentosas precoces adequadas para tratar as alterações metabólicas, com o objetivo de evitar o adoecimento ${ }^{3}$.

Além de elevar o risco de problemas cardíacos em 2,5 vezes, a presença dessa condição em trabalhadores jovens pode interferir em sua saúde, sua relação com o trabalho e seu modo de vida. Assim sendo, este estudo teve como objetivo estimar a prevalência de SM em colaboradores de uma fábrica de papel no Estado do Paraná.

\section{METODOLOGIA}

O estudo foi realizado dentro das determinações e dos padrões éticos previstos na Resolução no 196/96, do Comitê Nacional de Ética em Pesquisa (CONEP), e devidamente autorizado pela fábrica em questão.

Este é um estudo descritivo transversal, desenvolvido em uma fábrica de papel localizada no Estado do Paraná, doravante identificada apenas como fábrica. Para compor este 
estudo, foram utilizados os dados coletados no período de janeiro a dezembro de 2015, durante o exame periódico dos trabalhadores ativos, totalizando 1.787 colaboradores (1.643 homens e 144 mulheres), na faixa etária entre 21 e 65 anos.

Os colaboradores foram submetidos a exames laboratoriais como hemograma, glicemia, colesterol total e frações, triglicerídeos e gama GT, coletados por um laboratório certificado pela ABNT/INMETRO/NBR - ISO 9001/2000, contratado pela fábrica para realização dos exames periódicos. Os exames foram coletados no ambulatório da fábrica, com os colaboradores em jejum de, pelo menos, 12 horas. Os dados antropométricos foram avaliados pela equipe de enfermagem. Para realizar a aferição da pressão arterial, foi utilizado esfigmomanômetro da marca $\mathrm{BD}^{\circledR}$, calibrado, com os trabalhadores na posição sentada, com o braço apoiado sobre superfície firme e após 10 minutos de repouso.

A circunferência abdominal foi medida com o trabalhador em pé, sem roupa na região do abdômen, no ponto médio entre a borda costal e a cresta ilíaca, com fita métrica de $1,50 \mathrm{~m}$, graduada a cada $0,5 \mathrm{~cm}$, não distensível, porém flexível.

A estatura e o peso foram aferidos com balança antropométrica mecânica da marca Filizola ${ }^{\circledR}$, calibrada anualmente, com escala antropométrica com altura máxima de $2 \mathrm{~m}$ e graduação de $0,5 \mathrm{~cm}$ e capacidade máxima de $150 \mathrm{~kg}$. Para a determinação do peso e da altura, o colaborador permaneceu com roupa leve e descalço. $\mathrm{O}$ índice de massa corporal (IMC) foi calculado a partir do peso dividido pela estatura ao quadrado (IMC $=$ peso $(\mathrm{kg}) /$ [estatura $(\mathrm{m})]^{2}$ ). De acordo com o IMC, os trabalhadores foram classificados conforme os critérios publicados pela OMS em 2015: normal $\left(<25 \mathrm{~kg} \mathrm{~m}^{2}\right)$, sobrepeso $\left(25 \mathrm{a} 29,9 \mathrm{~kg} / \mathrm{m}^{2}\right)$, obesidade I (entre 30 e $34,99 \mathrm{~kg} / \mathrm{m}^{2}$ ), obesidade II (entre 35 e $\left.39,99 \mathrm{~kg} / \mathrm{m}^{2}\right)$ e obesidade III $\left(>40 \mathrm{~kg} / \mathrm{m}^{2}\right)^{1-3}$.

Para a análise estatística foi utilizado o teste do $\chi^{2}$ para avaliar se a incidência de SM está correlacionada com o gênero das pessoas avaliadas. $O$ teste de independência do $\chi^{2}$ avalia a existência de uma associação entre a variável de linha e de coluna em uma tabela de contingência construída a partir de dados da amostra.

\section{RESULTADOS}

O valor calculado do teste do $\chi^{2}$ foi igual a 14,352 , com 1 grau de liberdade. $O$ valor $p$ é menor que 0,0001 , permitindo concluir que há uma associação entre o gênero (linhas) e a ocorrência de SM (colunas) (Figura 1). No sexo feminino, foram encontrados 136 colaboradoras normais e 8 com SM, e no sexo masculino foram encontrados 1.349 colaboradores normais e 294 com SM.

O teste do $\chi^{2}$ avaliou se a incidência de SM está correlacionada com a faixa etária das pessoas avaliadas.

A Figura 2 apresenta o número de pessoas que foram devidamente analisadas. $\mathrm{O}$ valor calculado do teste do $\chi^{2}$ foi igual a 77,300, com 3 graus de liberdade. O valor p é menor que 0,0001 , permitindo concluir que há uma associação entre a faixa etária (linhas) e a ocorrência de SM (colunas).

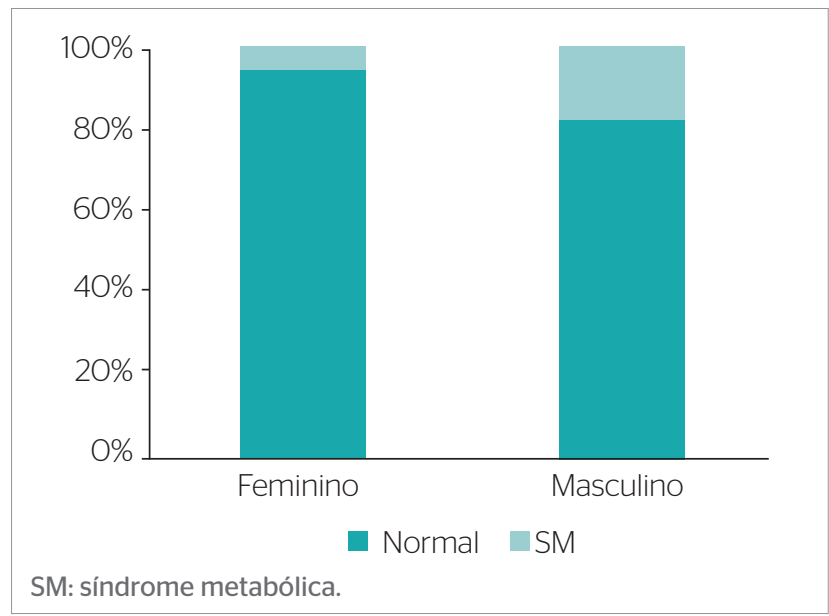

Figura 1. Incidência de síndrome metabólica nos sexos feminino e masculino de colaboradores de uma fábrica de papel no Paraná.

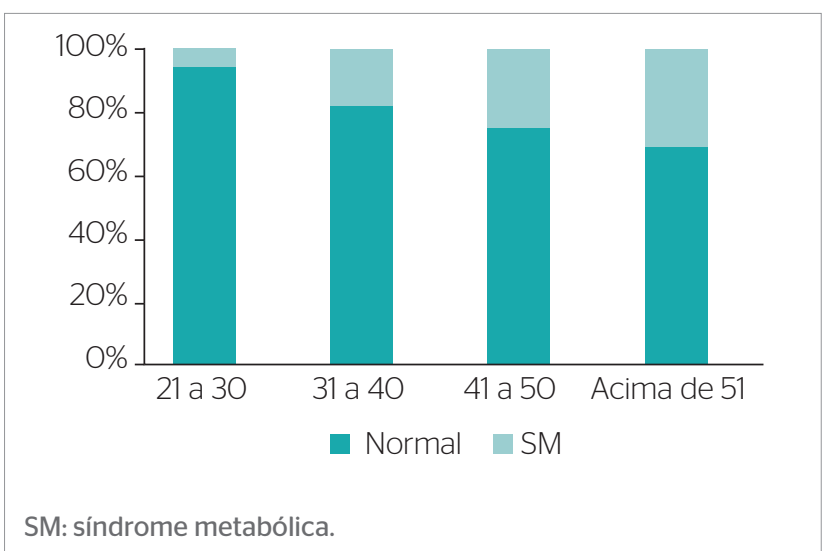

Figura 2. Incidência de síndrome metabólica por faixa etária dos colaboradores de uma fábrica de papel no Paraná. 
Quanto à faixa etária dos trabalhadores portadores de SM, $8 \%$ tinham de 21 a 30 anos, $19 \%$, de 31 a 40 anos, $26 \%$, de 41 a 50 anos, e 32\%, mais de 50 anos. Há uma correlação positiva entre a idade e a ocorrência de SM, a saber, quanto maior a idade, maior o percentual de SM, estabilizando (nesta pesquisa) na faixa de 50 anos (Figura 3). Os colaboradores das faixas etárias entre 41 e 50 anos e maior que 50 anos apresentam, estatisticamente, o mesmo nível de SM. Os colaboradores mais novos apresentam menor índice de SM quando comparados com os mais velhos $(p=0,0001)$.

\section{DISCUSSÃO}

Constatou-se que 16,9\% dos colaboradores da fábrica têm SM, sendo que a frequência diferiu entre os sexos, destacando-se maior índice de SM nos homens em comparação às mulheres.

Em estudos realizados em diversos países e no Brasil, identificou-se que a prevalência de SM varia de acordo com o sexo, a idade e a definição usada. Nos Estados Unidos, em uma pesquisa com 3.601 indivíduos, com idade variando entre 20 e 70 anos, a prevalência da SM foi de 34,5\% pelo conceito Adult Treatment Panel III (NCEP-ATP III), do National Cholesterol Education Program (NCEP) (33,7\% entre homens e $35,4 \%$ entre as mulheres), e de $39 \%$ pela IDF (39,9\% entre homens e $38,1 \%$ entre mulheres) ${ }^{5}$.

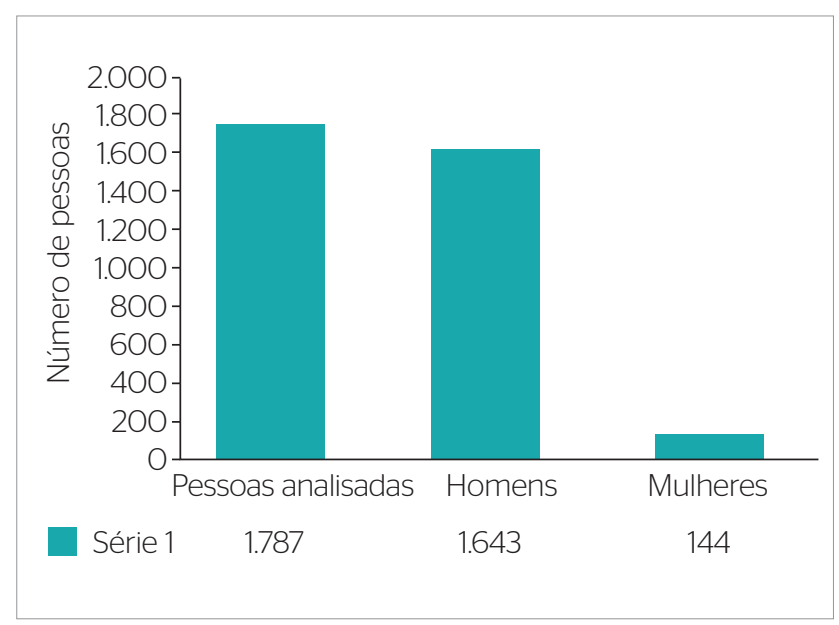

Figura 3. Número de pessoas analisadas.
Em Portugal, um estudo com 16.856 indivíduos mostrou uma prevalência da SM de 27,5\% (28,7\% entre mulheres e 26,0\% entre homens) pelo critério do NCEP-ATP III ${ }^{6}$. Em um estudo realizado com trabalhadores da área da saúde da Turquia, aplicando o critério do NCEP-ATP III, a prevalência da SM foi de 5,2\% entre mulheres e 12,7\% entre homens ${ }^{7}$. Em uma pesquisa catarinense realizada na cidade de Florianópolis com trabalhadores de um hospital universitário utilizando o critério da IDF, a prevalência da SM foi de 21,9\% entre mulheres e de 19,4\% entre homens ${ }^{8}$. Em Porto Alegre ${ }^{3}$, em um estudo transversal com 740 trabalhadores de um hospital universitário de grande porte, a prevalência total de SM foi de $12,8 \%$, sendo $16,2 \%$ do sexo masculino e $11,6 \%$ do feminino com o critério IDF. No Espírito Santo ${ }^{1}$, um estudo com o critério IDF realizado com policiais civis identificou SM em $16,5 \%$ dos participantes, sendo $18,6 \%$ entre mulheres e $15,8 \%$ entre homens. Na literatura, encontramos uma grande variação na prevalência da SM em trabalhadores, considerando o perfil da população estudada e a definição da SM adotada.

Esses dados mostram o quanto é importante a implantação de Programas de Qualidade de Vida, visando reduzir ou prevenir fatores de risco e a ocorrência de agravos. Esses programas promovem a melhoria da saúde em médio e longo prazo, o que proporciona melhores condições de trabalho e de vida.

A orientação sobre hábitos saudáveis e a realização de exames de rotina dos colaboradores são importantes para a manutenção da saúde ${ }^{2}$.

Apesar de existirem três linhas de alimentação na fábrica em questão (caseira, light e gourmet), uma nutricionista para elaborar o cardápio, Programas de Qualidade de Vida, como Ginástica Laboral, Programa Superando Medidas, Programa Antitabagismo, Programa Grupos de Controle (obesidade, hipertensão, diabetes) e Programa Álcool e Drogas, é necessária a conscientização do colaborador quanto ao problema e suas consequências para a saúde. Os hábitos de vida saudável, a escolha de alimentos saudáveis e a prática de atividade física regular devem ser estimulados, caso contrário haverá pessoas e trabalhadores cada vez mais medicados e com limitações físicas, principalmente entre os homens na faixa etária de 41 a 65 anos, na qual foi observada maior incidência de SM. 


\section{CONCLUSÃO}

A SM nos colaboradores da fábrica em questão foi afetada pelo sexo e pela idade, com maior incidência no sexo masculino e em pessoas da faixa etária de 41 e 65 anos. Esses trabalhadores precisam conhecer os riscos que envolvem sua condição e receber estímulos para cuidar da sua alimentação e fazer atividade física regular. A fábrica precisa continuar com seus Programas de Qualidade de Vida, mas agora com foco maior nesse público, fazendo com que tais trabalhadores participem mais dos programas após o conhecimento do seu problema.

\section{REFERÊNCIAS}

1. Molina MDCB, Morais AO, Saporiti AF, Salaroli LB, Boreges LH. Síndrome metabólica em profissionais de transporte de cargas especiais na rodovia do Espírito Santo. UFES Rev Odontol. 2008;10(4)37-47.

2. Felipe-de-Melo ERT, Silva RCR, Assis AMO, Pinto EJ. Fatores associados à síndrome metabólica em trabalhadores administrativos de uma indústria de petróleo. Ciênc Saúde Coletiva. 2011;16(8):3443-52.

3. Leite EB, Anchieta VC. Identificação de síndrome metabólica em policiais civis do Distrito Federal, Brasil. Brasília Med. 2013;50(3):186-93.

4. Rossa CLB, Caramori PRA, Manfroi WC. Síndrome metabólica em trabalhadores de um hospital universitário. Rev Port Cardiol. 2012;31(10):629-36.

5. Ford ES. Prevalence of the metabolic syndrome defined by the International Diabetes Federation among adults in the U.S. Diabetes Care. 2005;28(11):2745-9.
6. Fiuza M, Cortez-Dias N, Martins S, Belo A, Dias AD, Neves AS, et al. Síndrome Metabólica em Portugal: Prevalência e Implicações no Risco Cardiovascular - Resultado do Estudo VALSIM. Rev Port Cardiol. 2008;27(12):1495-529.

7. Oğuz A, Sağun G, Uzunlulu M, Alpaslan B, Yorulmaz E, Tekiner E, et al. Frequency of abdominal obesity and metabolic syndrome in healthcare workers and their awareness levels about these entities. Turk Kardiyol Dern Ars. 2008;36(5):302-9.

8. Gonzaga MLC. Prevalência de síndrome metabólica nos funcionários do Hospital Universitário da UFSC [Monografia]. Florianópolis: Universidade Federal de Santa Catarina; 2006.

Endereço para correspondência: Caími Tibiriçá de Carvalho - Fazenda Monte Alegre, s/n - Harmonia - CEP: 84274-000 - Telêmaco Borba (PR), Brasil -

E-mail: ctcarvalho@klabin.com.br 